\title{
Nueva especie de ostrácodo del Plioceno de la Depresión del Guadalquivir (S.O. de España).
}

\author{
A new Pliocene ostracod species from the Guadalquivir \\ Basin (SW Spain).
}

\author{
F. Ruiz ${ }^{1}$, M.L. González-Regalado ${ }^{1}$, M. Abad ${ }^{2}$ \\ 1 Departamento de Ciencias de la Tierra, Facultad de Ciencias Experimentales, Universidad de Huelva, España. Avda. 3 de \\ marzo, s/n. Centro de Investigación en Patrimonio Histórico, Cultural y Natural (CIPHCN). Email: urocythereis@gmail.com, \\ ORCID ID: http://orcid.org/0000-0002-7110-3133; http://orcid.org/0000-0002-2626-9142. \\ 2 Departamento de Geología, Facultad de Ingeniería, Universidad de Atacama, Chile. ORCID ID: http://orcid. \\ org/0000-0003-1930-2650.
}

\section{RESUMEN}

En este trabajo se describe e ilustra una nueva especie de ostrácodo, Cyprideis civisi nov. sp., procedente de sedimentos pliocenos de la Cuenca del Guadalquivir (S.O. de España). Esta nueva especie se caracteriza por su pequeño tamaño (longitud: < $820 \mu \mathrm{m}$; altura: $<460 \mu \mathrm{m}$ ) y por presentar valvas fuertemente reticuladas y un menor tamaño en comparación con otras de este género. Cyprideis civisi nov. sp. vivió en los sedimentos arenosos de un medio infralitoral, sometido a la acción de tormentas y de ocasionales flujos de aguas dulces. En este marco, esta especie formaba parte de la dieta de gasterópodos, como ponen en evidencia las huellas de bioerosión halladas en las valvas.

Palabras clave: Ostracoda; Cyprideis civisi; ontogenia; depredación; paleoambiente.

\section{ABSTRACT}

A new ostracod species from Pliocene sediments of the Gualdalquivir Basin (SW Spain), Cyprideis civis nov. sp., is herein described and illustrated. This new species is distinguished in having small size (length: < $820 \mu m$; height $<460 \mu \mathrm{m}$ ) and strongly reticulated valves and a small size in comparison with other species of this genus. Cyprideis civis nov. sp. inhabited in the bottom, sandy sediments of an infralittoral palaeoenvironment, subjected to occasional freshwater fluxes and storms. In this scenario, this species was part of the gastropod diet, as evidenced by the bioerosion traces found in the valves.

Keywords: Ostracoda; Cyprideis civisi; ontogeny; depredation; palaeoenvironment. 


\section{Introducción}

El género Cyprideis es uno de los más abundantes y extendidos en medios euhalinos, mesohalinos e incluso oligohalinos desde finales del Oligoceno y principios del Mioceno, especialmente en América, Europa y norte de África. En la actualidad, es un género frecuente en marismas (p.e., Martín-Rubio et al., 2004) y lagunas costeras (p.e., Ruiz et al., 2000) e incluso salinas (Bodergat et al., 1991). Sus caparazones y valvas pueden ser transportados por las corrientes y aparecer en la plataforma interna (Bonaduce et al., 1976).

En el Neógeno del sur de Europa, diversos trabajos se han centrado en el estudio de las especies de Cyprideis (p.e. Decima, 1962; Ligios \& Gliozzi, 2011) y de su origen (Gliozzi et al., 2017). En el este y sureste de España, el estudio de las asociaciones de ostrácodos ha permitido definir nuevas especies de este género (Van Harten, 1975) y, de forma especial, caracterizar la crisis de salinidad messiniense (Guerra-Merchán et al., 2010; Clauzon et al., 2015).

En este trabajo, se describe una nueva especie de Cyprideis en el Néogeno del suroeste de la Depresión del Guadalquivir, su evolución ontogénica final, las evidencias de depredación en sus valvas y caparazones, así como las condiciones paleoambientales en las que vivía.

\section{La Formación Arenas de Huelva: la sección de Bonares.}

La Formación Arenas de Huelva (Civis et al., 1987) es una de las unidades neógenas más representativas del sector suroccidental de la Cuenca del Guadalquivir. Su potencia varía entre los 15 y 25 m, con una base constituida por un nivel característico de limos verdes glauconíticos. A continuación se dispone un tramo de arenas amarillas finas y medias, ocasionalmente muy bioturbadas, en el que se intercalan entre 3 y 8 niveles tempestíticos (González Delgado et al., 1995). Su sección tipo se sitúa en la plaza de toros de la ciudad de Huelva, si bien existen otras secciones representativas de esta Formación en las localidades de Bonares, Lucena, Moguer o El Rompido (Fig. 1, A), entre otras.

La macrofauna está muy bien representada por más de 300 especies de bivalvos, gasterópodos y escafópodos, fuertemente concentrados en los niveles tempestíticos. La microfauna es muy abundante, con unas 1.000 especies reconocidas de foraminíferos planctónicos y bentónicos, ostrácodos y nanoplancton calcáreo, entre otros grupos (Sierro, 1984; GonzálezRegalado, 1989; Ruiz \& González-Regalado, 1996).

La sección de Bonares Km.8 tiene una potencia de $3,5 \mathrm{~m}$ y se encuentra ubicada en el Km. 8 de la carretera comarcal A-5001, que une las localidades de Bonares y Lucena del Puerto, en la provincia de Huelva. La Formación Arenas de Huelva está representada por una alternancia de arenas limosas finas levemente bioturbadas (sobre todo Ophiomorpha) y niveles tempestíticos de acumulación de macrofauna, con un espesor variable entre 10 y $60 \mathrm{~cm}$ (Fig. 1, B). El tramo superior de la sección $(30 \mathrm{~cm})$ pertenece a la Formación Arenas de Bonares (Mayoral \& Pendón, 1986) está constituido por unos $30 \mathrm{~cm}$ de arenas rojizas medias a gruesas, levemente bioturbadas y dispuestas mediante discordancia erosiva sobre la Formación Arenas de Huelva.

Esta sección y otras muy próximas ha sido objeto de numerosos estudios paleontológicos, centrados en bivalvos (Andrés, 1982), gasterópodos (González Delgado, 1983), foraminíferos bentónicos (González-Regalado, 1986) o icnofacies (Mayoral \& Pendón, 1986). Ruiz \& González-Regalado (1996) estudian los ostrácodos de esta sección, cuyas especies más representativas son Aurila convexa, Carinocythereis whitei, Celtia quadridentata, Cistacythereis caelatura, Cytherella vulgata, Cytheridea neapolitana, Falunia costata, Flexus triebeli, Hiltermannicythere sphaerulolineata, Miocyprideis sp. y Semicytherura sella (Fig. 1, B). Entre ellas, destaca la presencia de Cyprideis sp., objeto de reevaluación y análisis en este trabajo.

\section{Material y métodos}

Se han obtenido 12 muestras de la sección Bonares Km.8, separadas cada $0,25 \mathrm{~m}$ en la vertical (Fig. 1, B). De cada una de ellas se extrajeron $0,5 \mathrm{~kg}$. que fueron desagregados por el método clásico de agua oxigenada en medio alcalino con pirofosfato sódico. Este peso se levigó a través de un tamiz de $0,177 \mathrm{~mm}$ de diámetro de malla y el residuo fue secado a una temperatura constante de $60^{\circ} \mathrm{C}$. La observación de los ostrácodos se realizó mediante un microscopio estereoscópico binocular CITOVAL Jena, con micrómetro incorporado. 


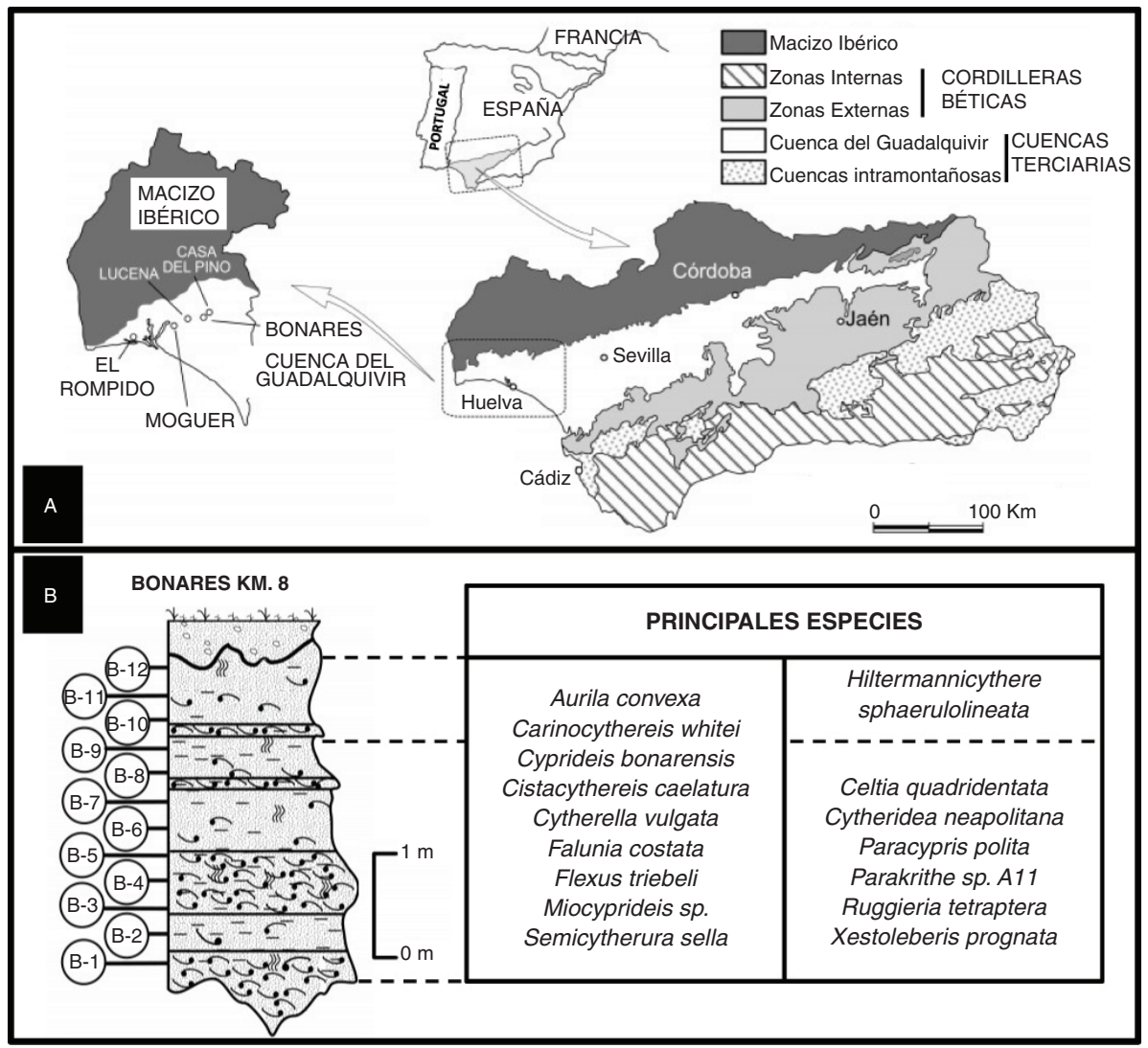

Fig. 1.-A. Situación geográfica y geológica de la sección de Bonares. B. Columna estratigráfica de la sección de Bonares, con la situación de las muestras y la distribución de las principales especies de ostrácodos (tomada de Ruiz \& González-Regalado, 1996).

Las fotografías electrónicas fueron realizadas en los Servicios Centrales de I+D de la Universidad de Huelva.

\section{Paleontología sistemática}

Clase Ostracoda LATREILLE, 1802

Orden Podocopida SARS, 1866

Familia Cytherideidae SARS, 1925

Género Cyprideis JONES, 1857

\section{Cyprideis civisi nov. sp.}

(Figs. 2-3).

1996 Cyprideis sp. Ruiz \& González-Regalado, 1996. Lám. 2, fig. 7.

\section{Etimología:}

In Memoriam del Dr. Jorge Civis, catedrático de Paleontología de la Universidad de Salamanca y gran impulsor de la Paleontología en el Neógeno de la Depresión del Guadalquivir.

\section{Localidad tipo:}

Sección de la Formación "Arenas de Huelva" situada en el Km. 8 de la carretera comarcal A-5001 que une las localidades de Bonares y Lucena del Puerto (Huelva, S.O. de España).

\section{Distribución:}

Plioceno Inferior. Solo se han encontrado ejemplares de esta especie en la localidad tipo.

\section{Holotipo:}

Una valva izquierda de una hembra adulta (M-0-1; Fig. 2, 1). Longitud: $780 \mu \mathrm{m}$; Altura: $431 \mu \mathrm{m}$. 


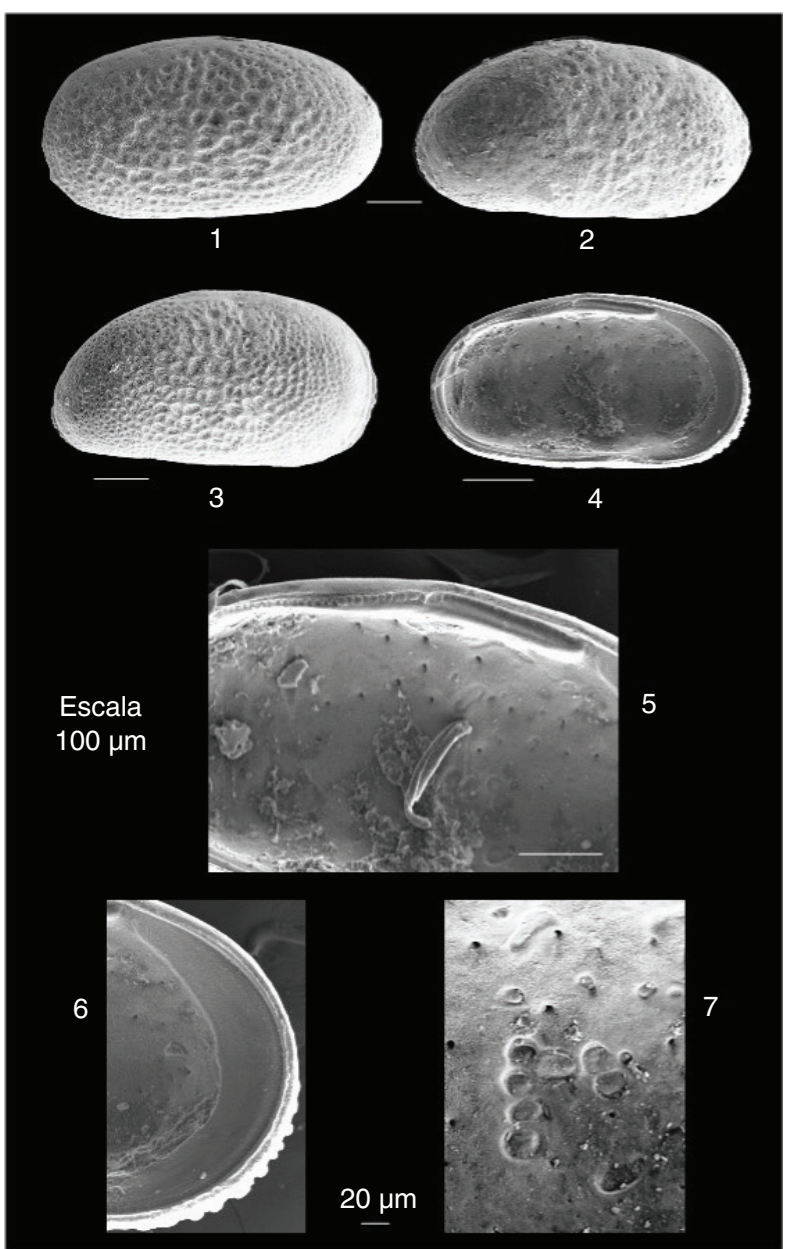

Fig. 2.-Cyprideis civisi nov. sp.. 1: Holotipo (M-0-1). Valva izquierda de una hembra; 2: Valva derecha de una hembra; 3: Valva derecha de un macho adulto. 4: Vista interna de una valva izquierda de una hembra; 5 : Detalle de la charnela de la figura 2.4; 6: Detalle del margen anterior interno de la Fig. 2.4.; 7 : Detalle de las impresiones de los músculos aductores.

\section{Alotipo:}

Una valva derecha de macho adulto (M-0-6).

\section{Paratipos:}

Dos valvas izquierdas de hembras adultas (M-0-2 y M-0-4), dos valvas derechas de hembras adultas (M-0-3 y M-0-5) y una valva derecha de macho adulto (M-1-1).

\section{Material:}

81 valvas y caparazones y 21 fragmentos de valvas, incluidos adultos y las últimas mudas juveniles.

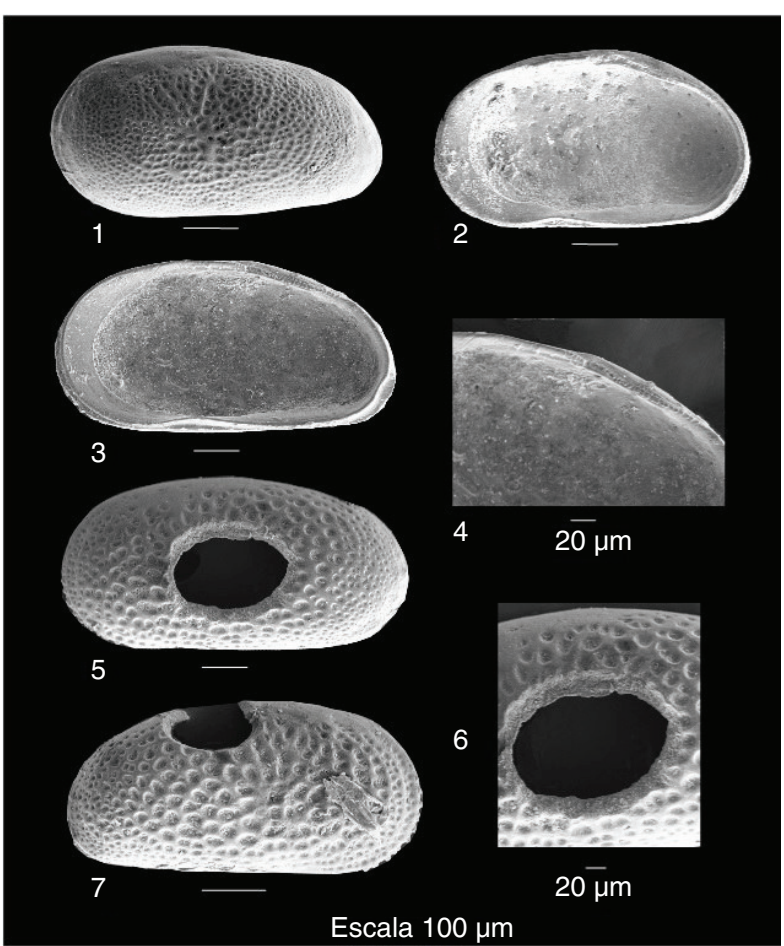

Fig. 3.-Cyprideis civisi nov. sp.. 1: Valva izquierda de una muda A-1 de macho; 2: Vista interna de una valva derecha de una muda A-1 de hembra; 3 : Vista interna de una valva derecha de una muda A-1 de macho; 4: Detalle de la charnela de la Fig. 3.3.; 5. Oichnus paraboloides en la zona central de una muda A-1; 6: Detalle de la Fig. 3.5.; 7. Oichnus paraboloides en la zona dorsocentral de una muda A-1.

La mayoría de ellas proceden de las muestras B-1 (23), B-2 (21), B-9 (9) y B-10 (13). En el resto, se han encontrado entre 1 y 4 valvas o fragmentos.

\section{Depósito:}

Laboratorio de Paleontología y Ecología Aplicadas, Facultad de Ciencias Experimentales, Universidad de Huelva.

\section{Diagnosis:}

Pequeño tamaño (longitud: $<820 \mu \mathrm{m}$; altura: $<460 \mu \mathrm{m})$ y una fuerte ornamentación externa, formada por retículos separados por gruesos muros. Esta reticulación se extiende a lo largo de la superficie de la valva, si bien es más patente en las zonas central y anterior del caparazón. Aparecen en filas subparalelas al margen en sentido dorsoventral. Esta ornamentación es más acusada en las valvas 
izquierdas que en las derechas, especialmente en las hembras.

\section{Caracteres generales:}

Valvas alargadas en sentido anteroposterior, con un borde anterior redondeado y denticulado (8-12 dentículos) y un borde posterior más agudo. Las hembras presentan un caparazón más subrectangular (Fig. 2, 1-2: longitud media: $810 \mu \mathrm{m}$; altura media: $430 \mu \mathrm{m})$, en tanto que los machos son algo menos alargados pero más aplanados, así como de menor tamaño (Fig. 2, 3: longitud media: $740 \mu \mathrm{m}$; altura media: $385 \mu \mathrm{m}$ ). La máxima altura de las valvas se sitúa en una posición levemente posterior en relación con el tubérculo ocular.

En vista interna, el borde ventral presenta un pronunciado doblez interno aproximadamente a 1/3 del borde anterior de las valvas. Este doblez, algo menos acusado, también está presente en el borde posterior. En las valvas izquierdas (Fig. 2, 4), los elementos principales de la charnela son un profundo surco subdividido en 10-14 fosetas en la parte anterior, seguido hacia la parte central por una barra subdividida en 26-30 dentículos, que precede a un surco posterior con 6-8 fosetas (Fig. 2, 5). Los canales marginales suelen estar bifurcados en el borde anterior.

Las impresiones de los músculos aductores son típicas de la familia y están conformadas por una fila de cuatro impresiones oblongas en disposición posterior en relación a una impresión arriñonada situada en la parte dorsal de esta fila, que a su vez puede estar subdividida por un fino muro en dos impresiones menores (Fig. 2, 7).

\section{Comparación con otras especies y géneros:}

Cyprideis civisi nov. sp. se diferencia básicamente de otras especies de Cyprideis en el gran tamaño de la reticulación externa y en su distribución por todas las áreas de las valva. Los retículos alcanzan un diámetro que duplica los observados en formas punteadas/reticuladas de Cyprideis torosa Jones. Tiene un caparazón más plano que Cyprideis baetica Van Harten en vista dorsal, un reticulado mucho más patente en la zona centro anterior de las valvas que esta especie, así como posee espículas cortas en el margen anterior y un borde anterior más redondeado. Los ejemplares adultos de estas especies suelen superar $1 \mathrm{~mm}$ de longitud, un 20\% mayores que los de Cyprideis civisi nov. sp.

Cyprideis pannonica (Mehes) presenta una ornamentación punteada, mucho menos acusada que Cyprideis civisi nov. sp., así como un borde posterior más subcuadrado. Cyprideis heterostigma (Reuss) difiere de esta última especie en la presencia de un profundo surco que recorre la superficie de las valvas desde la parte media del borde dorsal hasta la zona central de ellas, así como por la presencia de unas espinas mucho más agudas y alargadas en el borde anterior.

Cyprideis civisi nov. sp. guarda un parecido superficial con el género Miocyprideis Kollman, si bien presenta diferencias significativas con algunas de las especies neógenas más representativas de este género. Se diferencia de Miocyprideis italiana Moos por presentar una ornamentación basada en retículos regularmente repartidos por la superficie de las valvas. Esta especie está ampliamente representada en el Neógeno y Pleistoceno perimediterráneo (p.e. Temani et al., 2016).

\section{Depredación:}

Se han encontrado 4 evidencias de bioerosión que afectan a ejemplares adultos y a mudas A-1, sobre un total de 102 valvas y fragmentos de valvas $(3,9 \%)$. Todas ellas se concentran en la zona central (Fig. 3, 5-6) y dorsocentral (Fig. 3, 7) de las valvas, pudiendo llegar a afectar a las dos valvas del caparazón.

Tres de las perforaciones observadas atraviesan completamente las valvas y tienen un perfil subelíptico, con el diámetro mayor (hasta $340 \mu \mathrm{m}$ ) en sentido anteroposterior. Presentan una sección cónica, con un diámetro externo de perforación mayor que su diámetro interno y un borde biselado (Fig. 3, 6). Una cuarta perforación cónica, de menor tamaño $(40 \mu \mathrm{m})$ no llega a atravesar totalmente la valva.

\section{Análisis ontogénico:}

Se disponen de ejemplares adultos (Fig. 2) y de las tres últimas mudas juveniles (Fig. 3, 1-4). En este rango, el paso de una muda a otra o al estadio adulto final supone un crecimiento de 50-70 $\mu \mathrm{m}$ en longitud y 20-30 $\mu \mathrm{m}$ en altura (Fig. 4). Además, se observa un progresivo engrosamiento de los muros, que ocasionan la transición desde mudas finamente 
reticuladas a formas adultas con retículos menos definidos debido al ensanchamiento de los muros que los delimitan (ver Figs. 2-3).

\section{Aproximación paleoambiental}

\section{Batimetría}

Las asociaciones de bivalvos y gasterópodos presentes en la sección Bonares Km.8 sugieren la presencia de un medio infralitoral, con una profundidad no superior a los $30 \mathrm{~m}$ (Andrés, 1982;

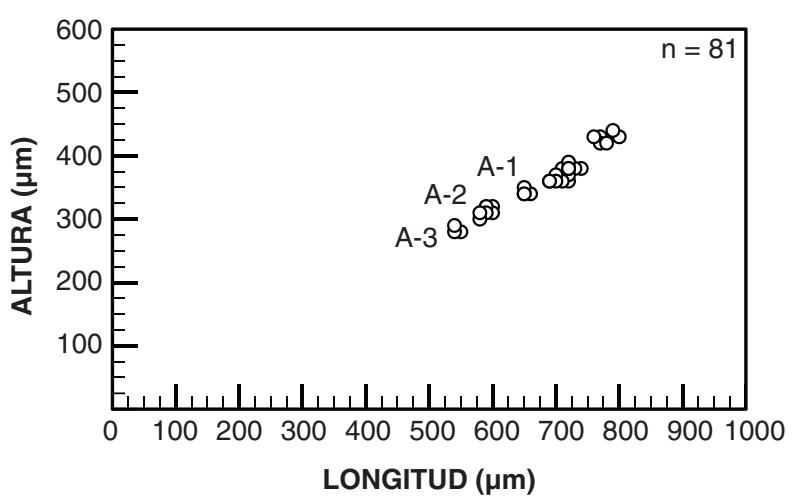

Fig. 4.- Relación longitud-altura en las últimas mudas y los adultos de Cyprideis civisi nov. sp.
González-Delgado, 1983). Esta profundidad disminuiría progresivamente hacia techo, dada la desaparición progresiva o disminución de la abundancia de ostrácodos de medios circalitorales internos e infralitorales externos (p.e. Hiltermannicythere, Falunia, Ruggieria). La asociación Aurila convexa-Carinocythereis whitei-Semicytherura sella, más abundante hacia el techo de la sección, se encuentra actualmente en medios muy someros $(<20 \mathrm{~m})$ del litoral de Huelva (Ruiz et al., 1997).

\section{Salinidad}

Ejemplares de Cyprideis civisi nov. sp. se han encontrado con valvas frecuentes de Miocyprideis $\mathrm{sp}$. Actualmente, especies de este género son abundantes en estuarios marinos sometidos a importantes variaciones de salinidad en función de los aportes fluviales, así como en las plataformas internas adyacentes (UNESCO, 1985; Dewi et al., 2007). Estas fluctuaciones también se reflejan en la presencia de ejemplares transportados de géneros dulceacuícolas, como Darwinula (Ruiz \& González-Regalado, 1996). Esta eurihalidad y proximidad de aportes fluviales también se refleja en las asociaciones de foraminíferos bentónicos y en las reconstrucciones paleoambientales de este sector (Fig. 5).

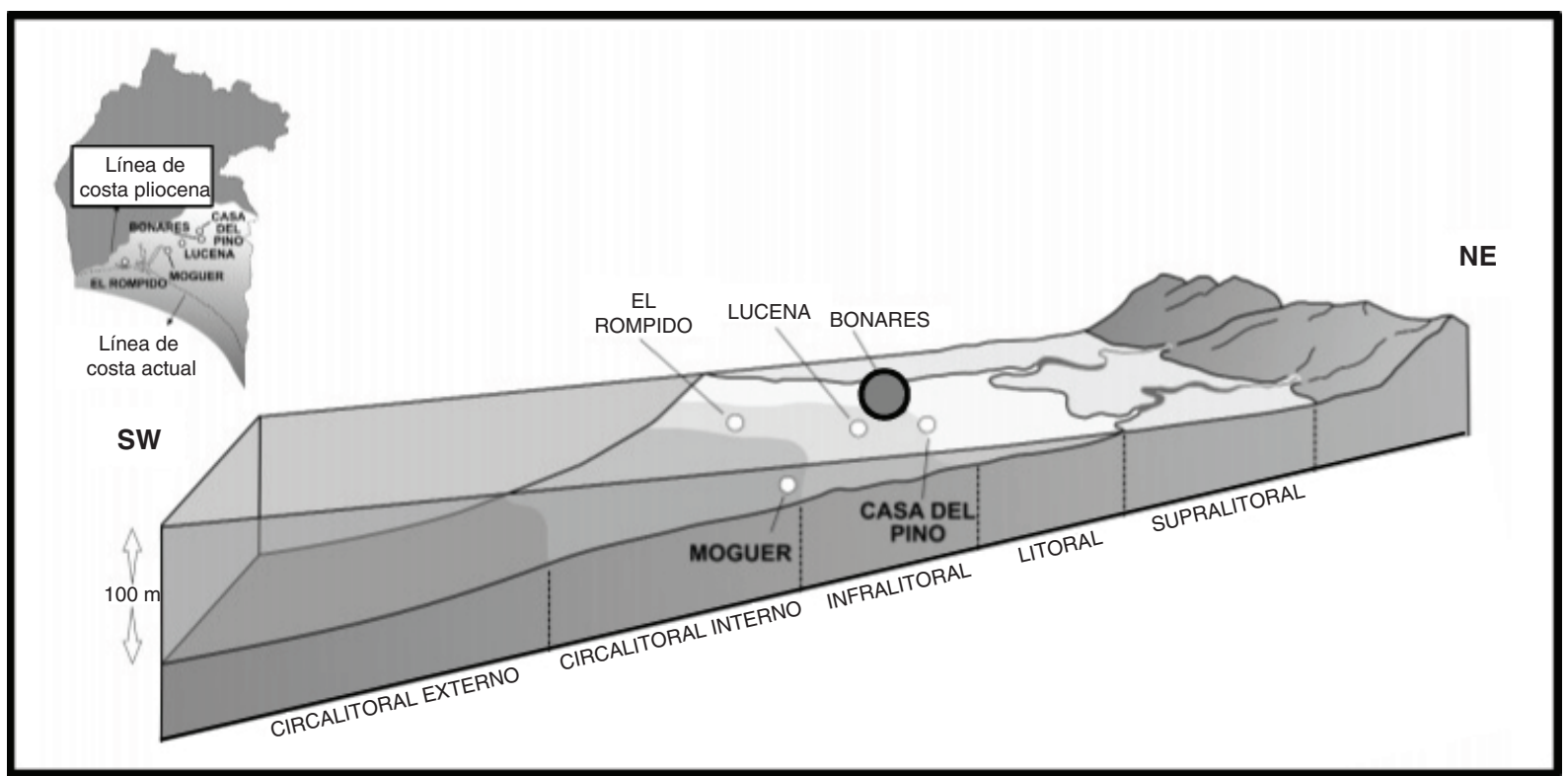

Fig. 5.-Reconstrucción paleoambiental del suroeste de la Depresión del Guadalquivir durante el Plioceno Inferior, con indicación de la situación inferida para la sección de Bonares (modificado de González-Regalado et al., 2009). 


\section{Temperatura}

El género Miocyprideis está bien representado en zonas costeras tropicales de África y Asia, tanto en la actualidad como en el Neógeno con temperaturas medias próximas a $24^{\circ} \mathrm{C}$ (UNESCO; 1985; Pais, 2012). En consecuencia, el medio sería algo más cálido que el actual litoral de Huelva (invierno: 13-14 ${ }^{\circ} \mathrm{C}$; verano: $21-24^{\circ} \mathrm{C}$ ) (Ruiz et al., 1997).

\section{Energía del medio}

El análisis tafonómico de los bivalvos presentes indica una energía baja en la base de la sección, que aumentaría hacia techo en función del grado de desarticulación y fragmentación de las valvas o la orientación de las mismas (Andrés, 1982). Esta transición progresiva se vería alterada por las tormentas que darían lugar a los niveles tempestíticos (González-Delgado et al., 1995).

\section{Depredación}

Las perforaciones observadas en las valvas de Cyprideis civis nov. sp. corresponderían a Oichnus paraboloides Bromley, un icnogénero que ha sido atribuido a la acción depredadora de gasterópodos y que en otros grupos también suele localizarse preferentemente en la zona central de las valvas (Lorenzo \& Verde, 2004).

\section{AGRADECIMIENTOS}

Con este trabajo, sus autores quieren expresar su agradecimiento, a título póstumo, al profesor Dr. Jorge Civis por las enseñanzas, disponibilidad, amabilidad de trato y profesionalidad mostradas hacia ellos a lo largo de más de 35 años de gratificante relación personal.

Agradecemos a D. Cristobal Cantero sus sugerencias y disponibilidad para la realización de las fotografías electrónicas. También se agradecen los constructivos comentarios realizados por dos revisores anónimos, que han contribuido a la mejora de este trabajo.

Este trabajo ha sido financiado por el Grupo de Investigación RNM-238 (Paleontología y Ecología Aplicadas) de la Junta de Andalucía, así como por el Plan Propio de Investigación de la Universidad de Huelva.

\section{Referencias}

Andrés, I. (1982). Estudio malacológico (Clase Bivalvia) del Plioceno marino de Bonares (Huelva). Tesis Doctoral, Universidad de Salamanca, $410 \mathrm{pp}$.
Bodergat, A.M.; Rio, M. \& Andreani, A. M. (1991). Composition chimique et ornementation de Cyprideis torosa (Crustacea, Ostracoda) dans le domaine paralique. Oceanologica Acta, 14: 505-514.

Bonaduce, G.; Ciampo, G. \& Masoli, M. (1976). Distribution of Ostracoda in the Adriatic Sea. Pubblicazione della Stazione Zoologia di Napoli, 40: 1-304.

Civis, J.; Sierro, F.J.; González-Delgado, J.A.; Porta, J. \& Valle, M.F. (1987). El Neógeno marino de la Provincia de Huelva: antecedentes y definición de las unidades litoestratigráficas. In: Paleontología del Neógeno de Huelva (Civis, J., ed.), Universidad de Salamanca, Salamanca, 9-21.

Clauzon, G.; Suc, J.-P.; Couto, D.D.; Jouannic, G.; Melinte-Dobrinescu, M.C.; Jolivet, L.; Quillévéré, F.; Lebret, N.; Mocochain, L.; Popescu, S.-M.; Martinell, J.; Doménech, R.; Rubino, J.-L.; Gumiaux, C.; Warny, S.; Bellas, S.M.; Gorini, C.; Bache, F.; Rabineau, M. \& Estrada, F. (2015). New insights on the Sorbas Basin (SE Spain): the onshore reference of the Messinian Salinity Crisis. Marine and Petroleum Geology, 66: 71-100. https://doi.org/10.1016/j. marpetgeo.2015.02.016

Decima, A. (1962). Ostracodi del genere Cyprideis Jones del Neogene e del Quaternario italiani. Palaeontographia Italica, 57: 81-133.

Dewi, K. T., Aryanto, N. C. D. \& Noviadi, Y. (2007). Land-sea interactions in coastal waters off NE Kalimatan: evidence from microfaunal communities. Bulletin of the Marine Geology, 22: 1-15.

Gliozzi, E.; Rodríguez-Lázaro, J. \& Pipik, R. (2017). The Neogene Mediterranean origin of Cyprideis torosa (Jones, 1850). Journal of Micropalaeontology, 36: 80-93. https://doi.org/10.1144/jmpaleo2016-029

González Delgado, J. A. (1983). Estudio de los gasterópodos del Plioceno de Huelva. Tesis Doctoral, Universidad de Salamanca, 474 pp.

González Delgado, J.A.; Andrés, I. \& Sierro, F.J. (1995). Late Neogene molluscan faunas from the North- east Atlantic (Portugal, Spain, Morocco). Geobios, 28: 459-471. https://doi.org/10.1016/ S0016-6995(95)80021-2

González-Regalado, M.L. (1986). Las asociaciones de foraminíferos bentónicos en las arenas fosilíferas del Plioceno de Huelva. Tesis Doctoral, Universidad de Sevilla, 314 pp.

González-Regalado, M.L. (1989). Estudio sistemático de los foraminíferos bentónicos de las arenas fosilíferas del plioceno de Huelva: su significado paleoecológico. Estudios Geológicos, 45: 101-119. https://doi. org/10.3989/egeol.89451-2485

González Regalado, M.L.; Ruiz, F.; Abad, M.; Civis, J.; González-Delgado, J.A.; Muñoz, J.M. \& García, E.X.M. (2009). Impact of storms on Pliocene benthic foraminiferal assemblages of southwestern Spain. Ameghiniana, 46: 345-360. 
Guerra-Merchán, A.; Serrano, F.; Garcés, M.; Gofas, S.; Esu, D. \& Gliozzi, E. (2010). Messinian Lago-Mare deposits near the Strait of Gibraltar (Malaga, Spain). Palaeogeography, Palaeoclimatology, Palaeoecology, 285: 264-276. https://doi.org/10.1016/j. palaeo.2009.11.019

Ligios, S. \& Gliozzi, E. (2011). The genus Cyprideis Jones, 1857 (Crustacea, Ostracoda) in the Neogene of Italy: a geometric morphometric approach. Joannea Geologie und Paläontologie, 11: 110-112.

Lorenzo, N. \& Verde, M. (2004). Estructuras de bioerosión en moluscos marinos de la Formación Villa Soriano (Pleistoceno tardío-Holoceno) de Uruguay. Revista Brasileira de Paleontologia, 7: 319-328. https://doi.org/10.4072/rbp.2004.3.03

Martín-Rubio, M.; Rodríguez-Lázaro, J. \& Pascual, A. (2004). Distribución areal y ecológica de las asociaciones de ostrácodos recientes en la marisma Victoria (Noja, Cantabria). Geogaceta, 36: 155-158.

Mayoral, E. \& Pendón, J.G. (1986). Icnofacies y sedimentación en zona costera. Plioceno Superior (?), litoral de Huelva. Acta Geologica Hispanica, 21-22: 507-513.

Pais, J. (2012). The Paleogene and Neogene of Western Iberia (Portugal). Springer, 139 pp. https://doi. org/10.1007/978-3-642-22401-0

Ruiz, F. \& González-Regalado, M.L. (1996). Les ostracodes du golfe mio-pliocène du Sud-Ouest de L'Espagne. Révue de Micropaléontologie, 39: 137-151. https://doi.org/10.1016/S0035-1598(96)90038-0
Ruiz, F.; González-Regalado, M.L. \& Muñoz, J.M. (1997). Multivariate analysis applied to total and living fauna: ecology of recent benthic Ostracoda off the North Cádiz (southwestern Spain). Marine Micropaleontology, 31: 183-203. https://doi.org/10.1016/ S0377-8398(96)00060-6

Ruiz, F.; González-Regalado, M.L.; Baceta, J.I.; Menegazzo-Vitturi, L.; Pistolato, M.; Rampazzo, G. \& Molinaroli, E. (2000). Los ostrácodos actuales de la laguna de Venecia (NE de Italia). Geobios, 33: 447-454. https://doi.org/10.1016/ S0016-6995(00)80078-9

Sierro, F.J. (1984). Foraminíferos planctónicos, bioestratigrafía y cronoestratigrafía del Mio-Plioceno del borde occidental de la Cuenca del Guadalquivir (S.O. España). Tesis Doctoral, Universidad de Salamanca, $391 \mathrm{pp}$.

Temani, R.; Nachite, D.; Sciuto, F.; Razgallah, S.; Bekkali, R.; Hayet, K. \& Gaaloul, N. (2016). Les Ostracodes plio-pléistocènes des séries sédimentaires de la bordure orientale du Cap Bon (coupe de l'Oued Lebna, Tunisie orientale). Carnets de Geologie, 18: 431-447. https://doi.org/10.4267/2042/61387

UNESCO (1985). L'estuaire et la mangrove du Sine Saloum. Rapports de I'Unesco sur les sciences de la mer, 32, 1-150.

Van Harten, D. (1975). Cyprideis baetica n. sp. (Ostracoda, Cytheridae) from the Miocene of southwestern Spain. Scripta Geologica, 32: 1-17. 